\title{
Una interpretación lógica sobre la estructura y el cambio urbano de la ciudad de San Juan de Puerto Rico
}

Dra. María del Carmen Zorrilla lassus (Universidad de Puerto Rico)

RESUMEN: SUMMARY:

En los albores del siglo XXI y en el ámbito geográfico del Caribe, la estructura urbana de San Juan de Puerto Rico refleja herencias culturales del pasado y transformaciones económicas y sociales reciente derivadas de la globalización.

San Juan aparece como una mancha urbana compuesta por varios municipios e integrada por

áreas diferenciadas desde los puntos de vista morfológico, social y funcional.

Su estructura y paisajes urbanos son el resultado de modelos culturales y sistemas productivos distintos que se han sucedido a través del tiempo sobre un mismo espacio. Hoy, la administración y los responsables del planteamiento se esfuerzan por corregir los problemas heredados, por resolver las carencias estructurales y por hacer de San Juan una aglomeración capaz de haer compatible desarrollo sostenible y calidad de vida de sus habitantes.
In the beginnings of the $X X I$ century and in the geographical environment of the Caribbean, the urban structure of San Juan from Puerto Rico reflects cultural inheritances of the past and derived recent economic and social transformations of the globalization.

San Juan appears like an urban stain composed by several municipalities and integrated by areas that differ greatly from the morphological, social and functional points of view. Their structure and urban landscapes are the result of cultural models and different productive systems that have been developing through time in the same space. Today, the administration and those responsible for the planning make an effort to correct the inherited problems, to solve the structural deficits and to make of San Juan a mass able to make compatible sustainable development and quality of their inhabitants' life. 


\section{INTRODUCCIÓN}

Puerto Rico es una isla localizada en los hemisferios norte y occidente, es decir entre los continentes americanos, sobre la línea del Ecuador. Su localización absoluta, o precisa, es de 18 grados norte y 67 grados oeste. Su composición territorial consiste de la Isla Grande, compuesta por 76 municipios, las isla municipios de Vioques y Culebra y una serie de islotes y cayos. La forma de la Isla es de un rectángulo con cuatro costas y su tamaño aproximado es de 100 millas de largo x 35 de ancho.

Puerto Rico es una isla tropical donde coexisten una variedad de ecosistemas tropicales, por lo que predomina la vegetación verde y exhuberante. La isla de Puerto Rico recibe una precipitación anual de lluvia que varía entre 30 y 200 pulgadas.

Uno de los fenómenos que más ha impactado a la sociedad puertorriqueña ha sido el crecimiento urbano que ha experimentado este pequeño territorio. La costa norte, otrora famosa por sus recursos costeros, hoy se ha convertido en una gran región metropolitana compuesta por trece municipios alineados de este a oeste teniendo a San Juan, la ciudad capital, como el eje central de la gran masa metropolitana.

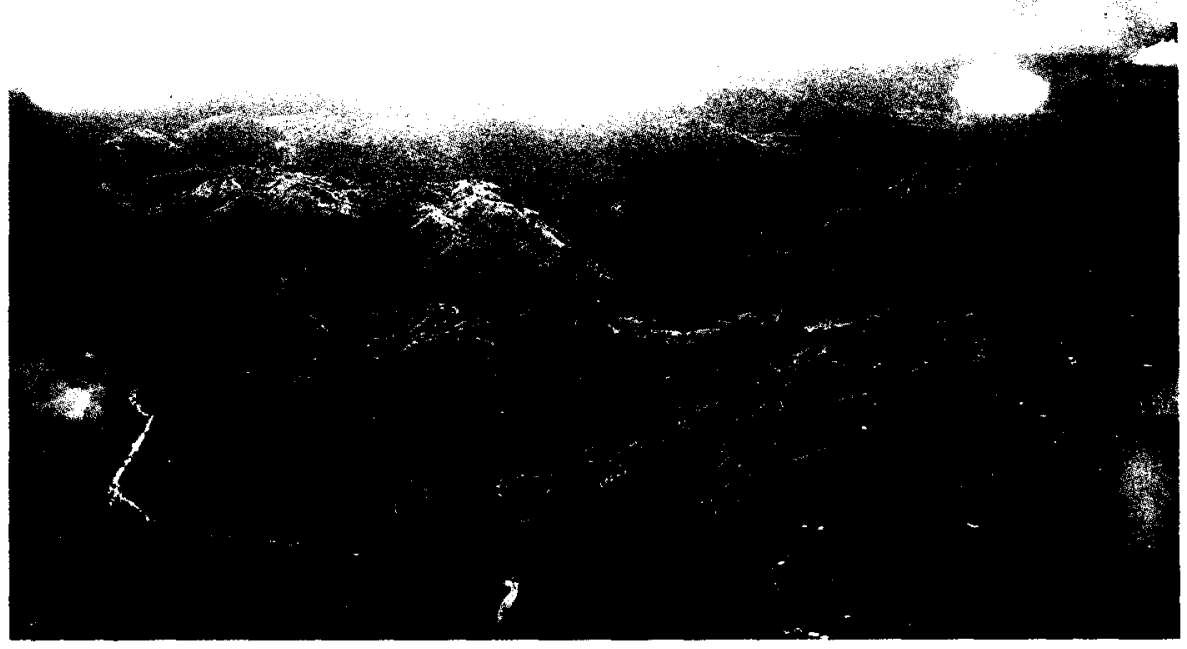

Figura 1. Cordillera Central 
El municipio de San Juan es parte integral del llano costero del norte, territorio que se extiende desde el río Arecibo al oeste, hasta el cabo de San Juan en el extremo noreste de la isla (PDOT). De acuerdo a la naturaleza geomorfológica, San Juan está dividido en tres zonas o bandas principales. Estas son la banda norte que incluye la la isleta de San Juan, el litoral norteño y el Caño Martín Peña. Tiene una serie de sistemas naturales pero entre ellos, el más importante, el Estuario de la Bahía de San Juan.

La segunda banda la constituye el llano costero que incluye la cuenca del Río Piedras y la quebrada Juan Méndez y entre sus sistemas naturales se encuentra el Jardín Botánico.

\section{La fundación del municipio y la sociedad de Sanjuanera}

Aunque el municipio de San Juan no fue el primer asentamiento a la llepgada de los españoles a Puerto Rico, tras el proceso de colonización, fue el municipio de mayor importancia en 1521. Una variedad de características hicieron de este municipio el eje central del crecimiento metropolítano. Entre ellas: su localización estratégica, sus características geográficas y sus recursos naturales y fisicos.

También fue importante la posición política que ocupó frente a los demás asentamientos, pues, se convirtió en la sede del gobierno central y en un centro financiero y comercial. Aunque los patrones de asentamientos de la Isla fueron continuos lo cierto es que muchos de los municipios fundados como Bayamón, Carolina, Trujillo Alto, Vega Baja y otros, se ubicaron en la periferia de San Juan en lo que hoy llamamos el área metropolitana.

Uno de los pueblos que se formó a las orillas de San Juan fue el pueblo de Rio Piedras, posteriormente adherido como territorio del Municipio. En un giro sorprendente luego de haber servido como un pueblo de producción e intercambio agrícola hoy es el primer centro de actividad universitaria y donde se encuentra la Universidad del Estado a la que hoy represento.

La población San Juanera es de aproximadamente 437.745 habitantes (censo 1990) en una extensión territorial de 47.8 millas cuadradas. EI $99.5 \%$ vive en la zona urbana y el $5 \%$ vive en las zonas rurales del municipio. En 1990 la densidad poblacional era 9,158 habitantes por milla cuadrada. A estas cifras se le añade que en la década de 1990 había una población flotante de 134.571 personas que llegan al municipio a trabajar. 


\section{La movilidad dentro del espacio}

Al comenzar el proceso de colonización los primeros asentamientos se establecieron en la áreas costeras y en las márgenes de los ríos como fue el caso del poblado de Caparra, fundado por Juan Ponce de León. Conforme fueron pasando los siglos y la Isla de Puerto Rico cobró mayor importancia para las metrópolis europeas los asentamientos posteriores se fueron localizando tierra adentro en busca de una mayor seguridad. Aún así, la costa norte fue el lugar preferido para los asentamientos. Caparra que había sido el centro de mando para la colonización en Puerto Rico tuvo poca duración trasladando así el centro entre 1519-1521 a lo que hoy conocemos como San Juan (Sepúlveda, 1989).

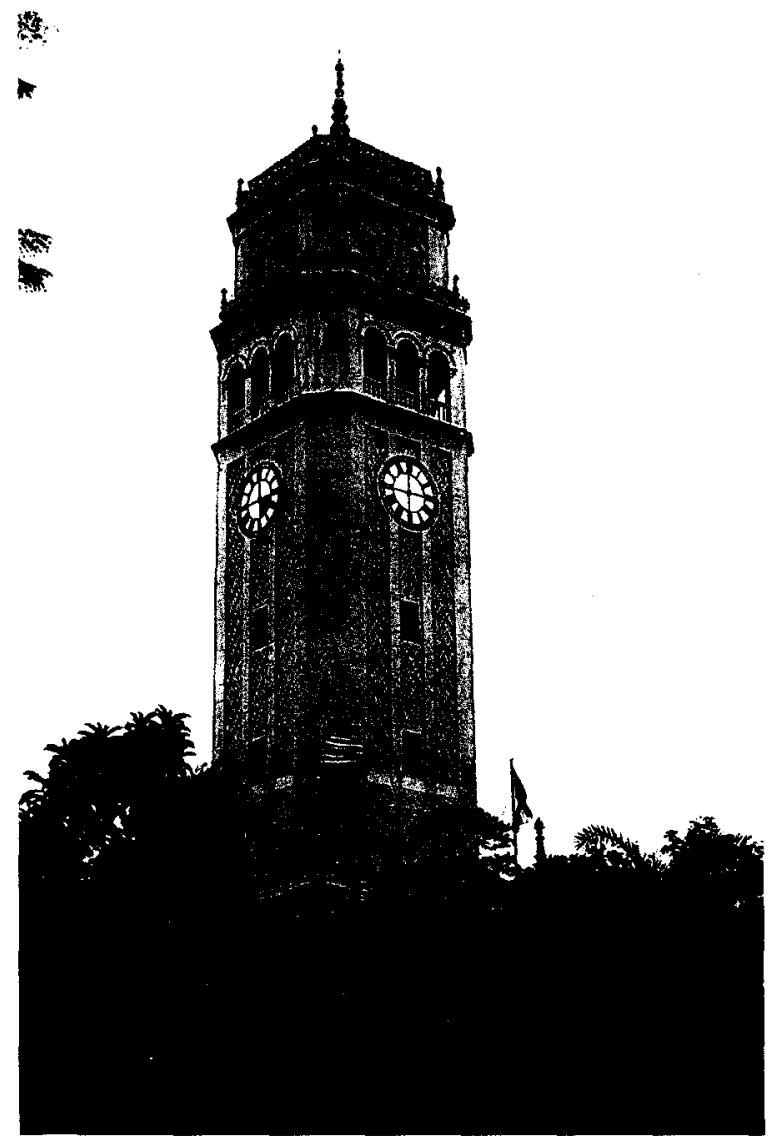

Figura 2. Universidad Estatal de Rio Piedras 
Otra razón de peso también para la búsqueda de nuevos asentamientos a partir del siglo XVIII fue el cambio de una economía de subsistencia a una agraria, donde no sólo la costa tenía importancia sino el interior montañoso el cual cobró importancia por la producción de un producto de gran acogida: el café.

Los pueblos costeros siguieron teniendo gran atractivo para los hacendados de la caña, ya que están formados por grandes extensiones de terreno propios para la siembra de la caña de azúcar que fue el producto de mayor importancia en la Isla hasta la segunda mitad del siglo XX, y que luego de la abolición de la esclavitud fue una de las mayores fuentes de trabajo.

No hay duda que para los que conocen un poco sobre el devenir histórico, político y económico de nuestro país estos factores fueron en gran medida determinantes en la organización y uso del espacio. Si bien se ha hablado mucho sobre la posibilidad de haber seguido un patrón de desarrollo urbano a partir de la colonización de América utilizando como parámetros las Ordenanzas Reales de Castillas (Sepúlveda 1989) o La Recopilación de las Leyes de Indias de 1573 en cuyo caso ninguna se ha podido comprobar con certeza, no se puede dudar de las semejanzas de la ciudad de San Juan con otras ciudades Andaluzas como las de Puerto Real en Cádiz. Según Hardoy (1975), el "carácter de las ciudades coloniales fue, en suma, una consecuencia del sitio natural y de los materiales empleados en su construcción. Las formas urbanas regulares adoptadas fueron soluciones eminentemente prácticas y funcionales en una empresa gigantesca como fue la conquista y colonización de América".

Al igual que San Juan otros pueblos de la Isla tienen un diseño ortagonal más o menos con una cuadrícula organizada o regular. En San Juan encontrarnos una plaza mayor excéntrica conocida como Plaza de Armas y otras plazas de menos importancia como la Plaza de Colón, la de San José y la desaparecida plaza del mercado. Alrededor de la Plaza de Armas encontramos edificios de funciones administrativas como fue y lo sigue siendo el ayuntamiento. Más adelante el crecimiento poblacional obligó a la ciudad militar a expandirse. Una vez comenzó esta expansión el diseño urbano perdió el patrón prevaleciente dominando quizás la desorganización o la irregularidad.

A partir del siglo 20 Puerto Rico experimentó un cambio urbano ascendente y vertiginosos. El país tuvo una economía agrícola con altas y bajas dentro del mercado internacional, eventos históricos como los del inicio del siglo 20: la crisis del mercado del 1912, la de los precios en 1920, la devastación de los huracanes San Felipe y San Ciprián y la Depresión de 1929 
(Baralt, 1993) fueron nefastos y determinantes para el desarrollo urbano de Puerto Rico. Tras años de búsqueda de alternativas de fianciamiento para la industria agrícola, el comercio del azúcar así corno sus industrias satélites declinaron inexorablemente. El azúcar dejó de ser la médula de la economía puertorriqueña (Baralt, 1993). La economía industrial adquirió una nueva jerarquía y años más tarde reemplazó a la economía agrícola. La industria del aguja y la construcción tomaron un nuevo auge.

\section{La ciudad como fuente de empleo}

San Juan siempre fue un lugar atractivo para los que buscaban un mejor futuro. En la primera mitad del siglo hubo una movilidad sustancial como consecuencia de algunos de los eventos históricos ya comentados. En esta primera mitad la población aumentó de 25.000 habitantes a 170.000, (Baralt, 1993) creando a su vez un problema de vivienda. Asi se fueron estableciendo los arrabales en la periferia de la ciudad murada la cual tenía graves problemas de espacio para vivienda y problemas en la infraestructura.

En la segunda mitad del siglo 20 , en términos comerciales, el municipio de San Juan, además de ser la ciudad capital, el principal centro turístico y político se convirtió en centro de empleo donde se concentran la mayoría de las agencias públicas y empresas privadas del país. En San Juan se concentra el mayor volumen de empleos de todo Puerto Rico. En el 1992 el $44 \%$ de los empleos se concentraban en el municipio (PDOT), también se encuentra el $47 \%$ del total de empleos del sector turístico, el $44 \%$ del gobierno, el $42 \%$ en el área de servicios, el $40 \%$ en la construcción y el $36 \%$ del comercio.

\section{EL IMPACTO DE NUEVOS MODELOS}

\section{En el cambio político}

Hasta finales del sigio 19 la organización territorial del país respondió a los patrones heredados por la Metrópoli Española, bien fuera por algunos de los estatutos de las Leyes de Indias referentes al ordenamiento territorial, por la experiencia que traían los colonizadores y por las condiciones fisicas y naturales de la Isla. Como dije anteriormente la mayoría de los pueblos de la Isla están organizados en torno a la cuadrícula donde la plaza y la iglesia católica ocupan un lugar prominente. Según los 


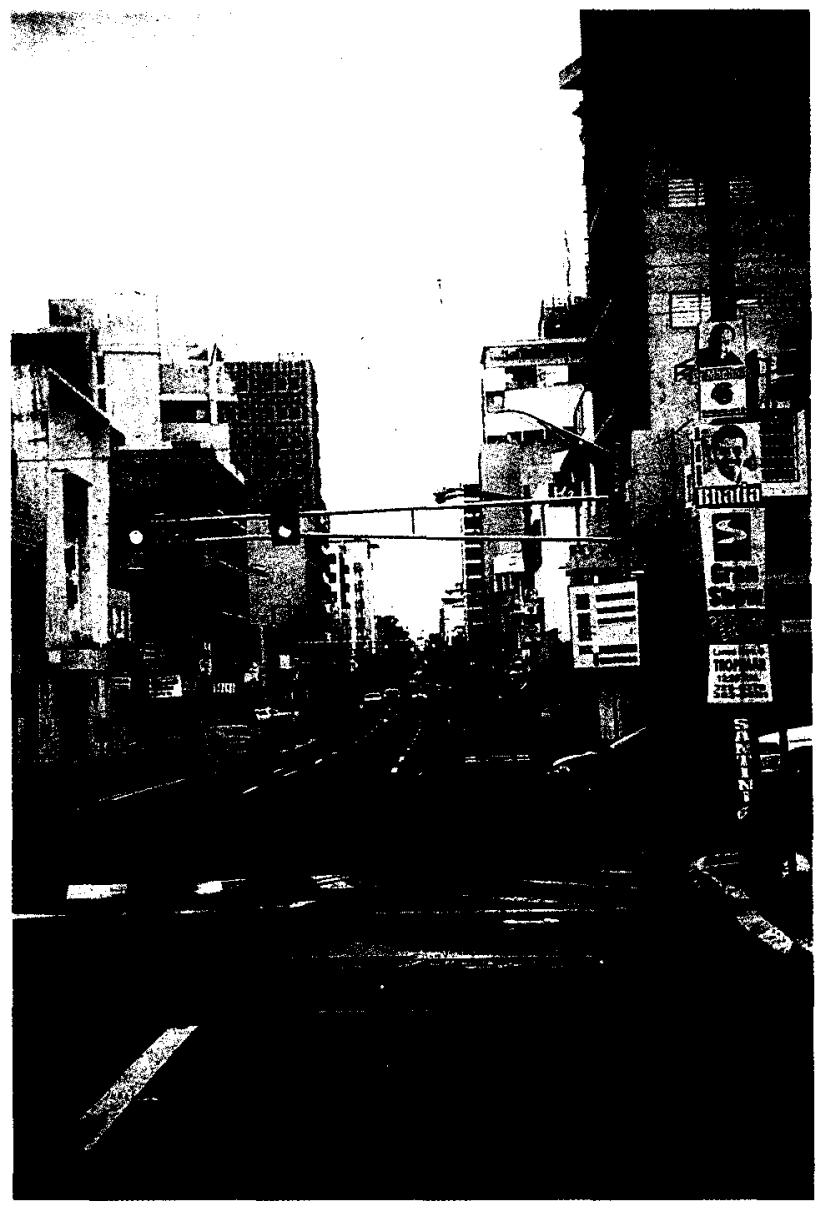

Figura 3. Avenida Ponce de León. Santurce (San Juan)

municipios fueron creciendo se añadieron los ensanches y posteriormente en el siglo 20 las urbanizaciones.

En los inicios del siglo 19 uno de los organismos responsables de la organización del espacio fue la Junta de Hacendados y Comerciantes, mejor conocida como la Junta de Caminos. Un elemento importante en la organización del espacio fue la construcción de vías de comunicación las cuales permitieron asentamientos alineados en torno a las vías o carreteras. Uno de los casos más importantes fue la construcción del 
Camino Real hoy conocido como Avenida Ponce de León y el camino de Bayamón a Cataño. Estas vías permitieron un mayor flujo entre los pueblos aledaños.

A partir del siglo 20 tras la guerra Hispano-cubanoamericana, la isla de Puerto Rico pasó a manos de los Estados Unidos, evento que se reflejó rápidamente en la morfología y estructura urbana del país. Aunque los cambios urbanos se dieron en concordancia de lo estipulado por el gobierno federal de los Estados Unidos no fue hasta el 1942 que se creó la Junta de Planificación de Puerto Rico, entidad responsable de regular el uso y desarrollo de los terrenos. A partir de ese momento los instrumentos que regulaban el suelo fueron el Plan de Uso de Terrenos de la Región Metropolitana de San Juan de 1982 y el reglamento de Zonificacición. Otra entidad que apareció fue la Administración de Reglamentos y Permisos conocida por sus siglas (ARPE). Tras vario años, en 1991, se aprobó la Ley de Municipios Autónomos, la cual facultá a los municipios de ejercer un rol central en el desarrollo urbano, social y económico. Esta ley permitió el uso de un nuevo instrumento llamado Plan de Ordenamiento Territorial. Estos movimientos trajeron nuevos usos de terrenos y nuevos protagonistas en la organización del territorio.

\section{En el cambio económico}

El modelo económico de Puerto Rico fue otro factor que ha determinado la organización del territorio. Como todas las economías de los paises, la de la Isla ha estado a merced de los cambios y fluctuaciones internacionales, así como a una economía de dependencia colonialista, primero de España y luego de Estados Unidos. De una economía de subsistencia pasó a una economía agraria, luego a una industrial y posteriormente a una economía de manufactura y tecnología.

Una vez fragmentada la economía en la segunda mitad del siglo 20 , el pais se sumió en una gran pobreza. Es a final de las décadas del 40 y a principios del 50 que se empieza a dar una segunda movilidad social del campo a la ciudad en busca de trabajo. Ya para los primeros años del 1950 comienzan a surgir en la estructura urbana de San Juan unas bolsas de pobreza situadas en las áreas pantanosas. También la ciudad que había comenzado a crecer extramuros debido a la falta de espacios se fue ampliando más allá de la isleta. Se construyeron viviendas en Santurce y Hato Rey por personas de dinero. Estas áreas, en su momento, se conviertieron en grandes zonas comerciales hoy conocidas como el Condado y Miramar . 


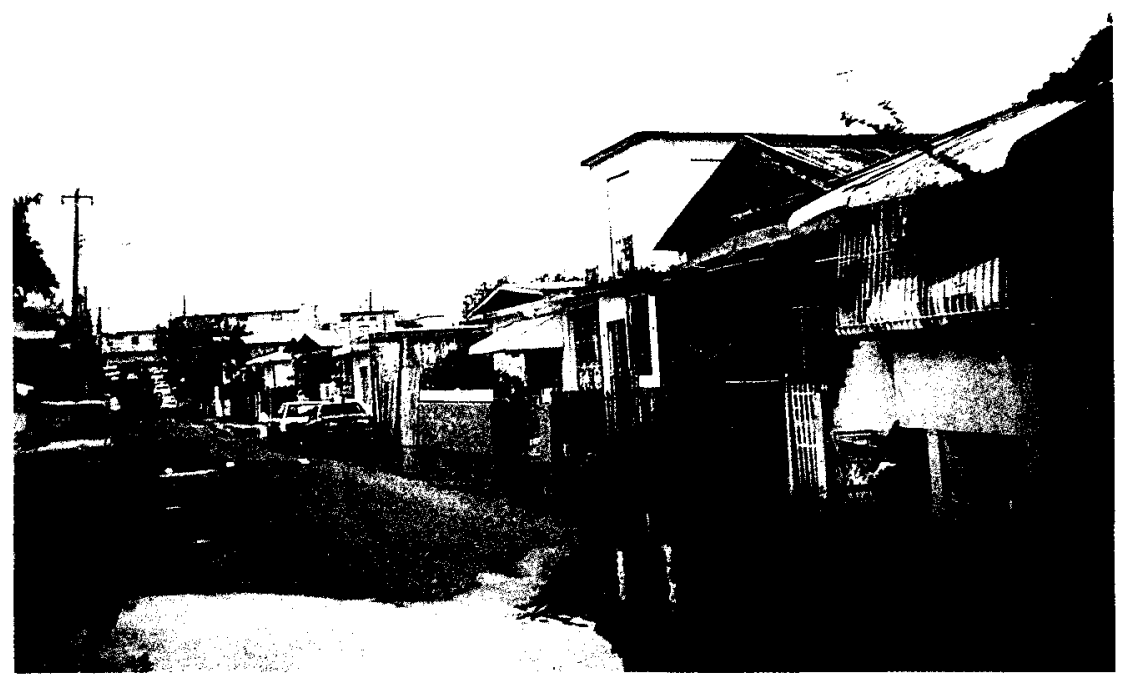

Figura 4. Barrio obrero de Santurce (San Juan)

En el cambio social

Una vez más el cambio de una economía agrícola a una industrial dio un giro a la organización y uso del espacio. La industrialización, el comercio y los servicios fueron las nuevas fuentes de empleo, localizadas en el área metropolitana por lo que la población de nuevo se movió en busca de oportunidades. Así los pobres y los ricos comenzaron a darle otra forma urbana al municipio de San Juan.

Aparecieron nuevas formas de subsidio para la construcción de vivienda económica, por lo que se generó nuevas fuentes de empleo en la construcción y surgieron nuevas formas urbanas como las barriadas obreras, las urbanizaciones de Roosevelt y Puerto Nuevo, esta última no fue la primera pero si la más grande en su clase. La tipologia de las casas se masificó y comenzarnos a ver grandes área urbanas homogéneas ocupadas por las clases proletarias.

Otro modelo nuevo para la Isla fue la construcción de viviendas públicas subsidiaria por el gobierno llamadas en un principio Caseríos, luego Residenciales públicos. Entre ellos unos de los más grandes. El residencial Nemesio Canales, San José y Lloréns Torres. Con el transcurrir del 
tiempo estas tres bolsas de pobreza se convirtieron en grandes centros aislados de la trama urbana donde la criminalidad se hizo patente y manifiesta. Hoy día como parte del plan de ordenamiento territorial se intenta integrar a estas comunidades al resto de la ciudad a través de diversos programas de rehabilitación fisica, económica y cultural.

Uno de los aspectos que se modificó en el municipio fue la movilización de las familias que habitaban las comunidades aisladas. Estos territorios fueron absorbidos prácticamente por el crecimiento urbano, por lo que los llamados arrabales se demolieron reubicando a las familias bien fuera en los residenciales públicos o mediante el programa de subsidio de vivienda en otras urbanizaciones de bajo costo. Arrabales inmortalizados en la literatura puertorriqueña fueron el llamado Fanguito y la Perla. Hoy sólo queda en el recuerdo el primero y el segundo perdura como testigo silente de aquellos terribles años.

La Perla, la cual se sitúa en la periferia de la ciudad murada, ha permanecido con más fuerza política y cultural posiblemente más que otras menos numerosas. Sin embargo, en un afán del gobierno por darle un carácter nuevo a estas comunidades, se les ha dado una nueva categoría de "comunidades especiales", en las cuales se invierte una gran cantidad de dinero para la recuperación de las mismas de manera que puedan descentralizarse del gobierno y puedan participar del proceso de organización y orden territorial como es el caso de la Península de Cantera.

\section{En el cambio tecnológico}

La Isla del Encanto no está al margen de la globalización. Las nuevas tecnologías se hacen patentes en los nuevos medios de producción. La industria farmacéutica así como la de tecnología y las comunicaciones son tres de las fuentes que generan la mayor cantidad de ingresos para la Isla. Empresas destinadas a la fabricación de medicamento así como a la fabricación de microfichas ocupan gran parte del territorio antes destinado para la siembra de caña, es decir parte de los llanos costeros.

\section{De frente al nuevo milenio}

La descentralización de los municipios se hace cada día imprescindible. Los planes de ordenamiento territorial le han dado las herra- 
mientas necesarias para hacerlo. La ciudad de San Juan es una ciudad con una serie de problemas heredados del pasado pero con una mayor concentración de profesionales conscientes de la necesidad de reorganizar el espacio y el orden público. Sin duda alguna, la función de las universidades y de las agencias medioambientales no se puede menospreciar, pues cada dia hay una mayor conciencia sobre los procesos de ordenamiento territorial y sobre sus usos. La aparición de nuevos proyecto con el fin de hacer de San Juan una ciudad moderna que compita en el mercado global se pone de manifiesto. La participación de un grupo de ciudadanos en la solicitud de la sede de las Olímpiadas del 2004 fue un ejemplo de las posibilidades de presentación que tenía la ciudad de San Juan como una ciudad moderna capaz de suplir las necesidades más exigentes de las grandes ciudades como la infraestructura, las dotaciones y equipamientos, los servicios, las comunicaciones y el quehacer cultural.

Los nuevos proyectos del gobierno municipal aspiran a desarrollar una ciudad vital, segura y limpia, sede de intercambio cultural y económico y promotora de la convivencia armoniosa entre sus ciudadanos. Se busca, a través de los planes de ordenamiento, fomentar un desarrollo urbano que no antagonice con los preceptos de conservación y preservación.

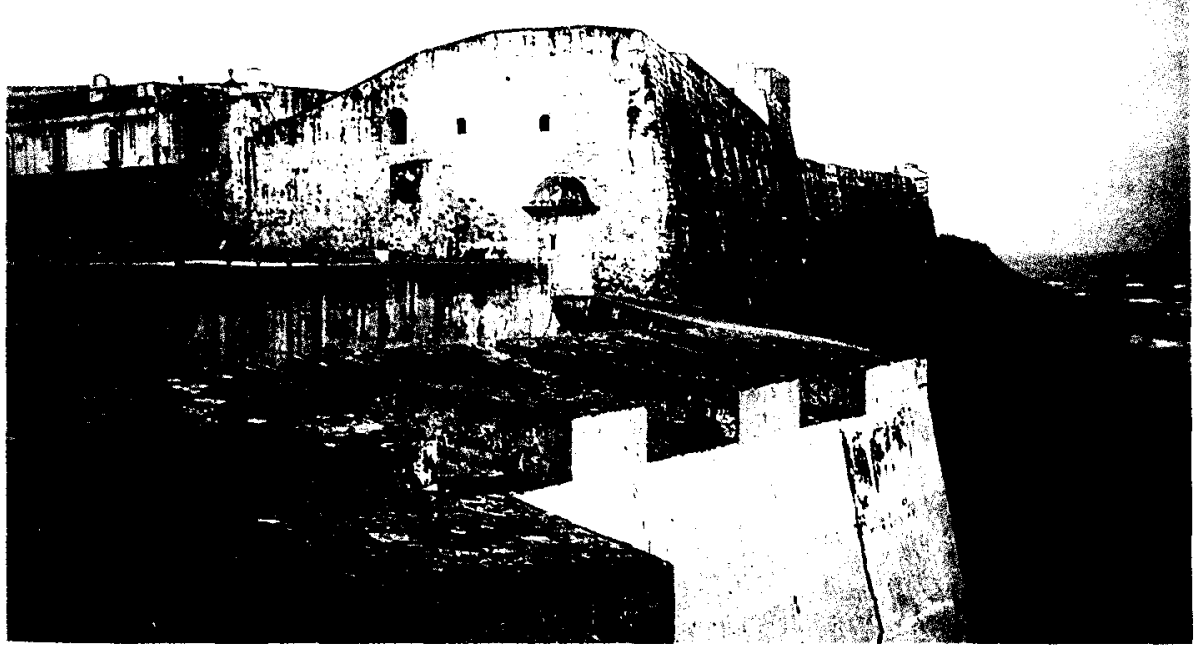

Figura 5. Viejo San Juan. Castillo de San Cristóbal 
Quizás entre todas las metas y objetivos más importantes se encuentra la participación ciudadana en relación a la toma de decisiones de aquellas acciones que impliquen un cambio en el uso del terreno que atente contra la seguridad y el bienestar de las comunidades. Este ha sido el caso de la intención de demolición de un complejo turístico de gran interés histórico, conocido como el Condado Trío, por parte del gobierno estatal y que la comunidad sanjuanera ha detenido con su militancia utilizando los mecanismos legales apropiados

\section{LA MORFOLOGÍA URBANA}

\section{La herencia española y anglosajona}

El aspecto fisico de la ciudad de San Juan refleja, fundamentalmente, la intervención cultural y urbana de dos pueblos. Por un lado, los cascos urbanos tradicionalmente coloniales heredados de España y, por otro lado, las grandes urbanizaciones con modelos de viviendas masificados como es el caso de las urbanizaciones estadounidenses.

El casco urbano de San Juan se manifiesta a través de sus calles adoquinadas y sus casas pegadas unas de otras construidas en su mayoria de mampostería. El territorio está organizado a partir de su plaza mayor y de sus iglesias como la Catedral y la Iglesia San José. El comercio está ubicado en las vías principales y las viviendas en las vías restantes. Su organización realza los hitos históricos.

Sin embargo, al salir de las murallas, San Juan se presenta como una ciudad amalgamada. Por un lado, decenas de urbanizaciones desparramadas por toda la región central del municipio construídas en su mayoría de hormigón con un solar de acuerdo con las posibilidades económica que ocupan los habitantes dentro de la escala social, y avenidas que sirven como corredores comerciales. De otro lado, centros o nodos financieros y comerciales organizados en torno a edificaciones verticales de gran tamaño. Todos estos nodos financieros y comerciales están entretejidos a través de la red vial. La autopista en estos momentos es el eje central de la Isla y las otras avenidas de primer orden sirven como elemento unificador entre los municipios de la región metropolitana.

Sin embargo, el crecimiento urbano de San Juan hacia el sur se dio emninentemente en función de la utilización del automóvil y no de los medios de transportación colectiva. Eso significa que cualquier habitante ne- 
cesita de un transporte individual para tener mayor movilidad dentro de la ciudad y poder utilizar diversos servicios que en ella se ofrecen como los centros comerciales.

\section{LA COMPOSICIÓN URBANA}

\section{Las área residenciales}

Como dije anteriormente las áreas residenciales no están concentradas en un área, sino todo lo contrario, se perciben completamente desparramadas. El crecimiento horizontal se da desde la franja norte hasta llegar a la periferia rural en el sur. De aqui que el concepto del suburbio norteamericano se haya adaptado a la realidad puertorriqueña. Es en esta periferia urbano-rural donde se concentran la mayor cantidad de casas de grandes extensiones de terreno, muy lujosas y que pertenecen a una clase social compuesta en su mayoría por abogados, médicos y comerciantes. Estas viviendas cuentan con un acceso controlado a las vías de rodaje del núcleo urbano por medio de un sistema de seguridad privatizado que pagan los residentes. Para tener acceso a estas viviendas se hace indispensable la utilización del automóvil ya que la transportación pública es limitada.

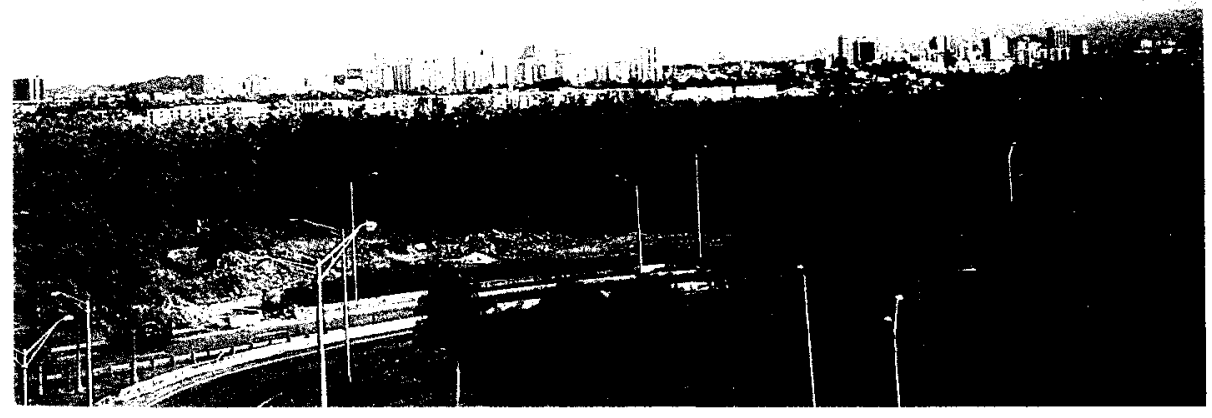

Figura 6. Hato Rey 
La otra franja residencial y la más numerosa se encuentra en la zona central del municipio y pertenece a la clase media, en la mayoría trabajadores gubernamentales o técnicos y obreros. Estas áreas no cuentan con acceso controlado y las parcelas y las superficies de construcción son más limitadas. Estas zonas integran los nodos financieros y comerciales y cuentan con mayores servicios de transporte público aunque no de gran eficiencia. Me refiero a las áreas de Hato Rey, Rio Piedras, Puerto Nuevo y Caparra.

\section{Las áreas comerciales}

Las área comerciales tanbién están ubicadas en diferentes nodos. La más importante que era el casco de San Juan perdió prominencia al aparecer los grandes centros comerciales. El primero y el más conocido es Plaza Las Américas el cual cuenta no solo con tiendas si no que ofrece una gran variedad de servicios en el área de la salud y las diversiones. A este se suman otros centros comerciales de gran teanaflo cerca de las área peri-urbanas de mayor poder adquisitivo Rio Piedras sur y Guaynabo.

\section{Las áreas industriales y las dotaciones}

La industria en Puerto Rico se ubica en función del uso de los suelos. El área industrial en el municipio de San Juan es limitada y se da en función del intercambio comercial a través de los puertos. Cuenta San Juan con dos grandes áreas de puertos marítimos concentrados ambos en uno de los espacios naturales más importantes de San Juan: el Estuario de la Bahía de San Juan. Uno de los puertos de interés turístico y comercial se encuentra al norte de la Bahía de San Juan y el otro hacia el sur de la Bahía conocido como Pueblo Viejo.

Por otro lado, el aeropuerto se encuentra en uno de los municipios aledaños de San Juan que es Carolina, en un eje entre los puertos marítimos y aéreos de la región.

Otras dotaciones lo son las unidades de salud pública y los hospitales privados que se encuentran a través de todo el territorio municipal. Aunque existe un nodo de salud que es el Centro Médico, una ciudad universitaria y de servicios médicos posiblemente de los más especializados. La mayoría de los residentes del municipio utilizan la medicina privatizada a través de siete hospitales.

En relación a las áreas recreativas, San Juan cuenta con tres parques de recreación pasiva: El Parque Muñoz Rivera, Muñoz Marín y el 
parque Central a través de una parte del Estuario de la Bahía de San Juan. La mayoría de las urbanizaciones cuenta con centros recreativos, algunos en muy mal estado. El municipio dispone también de un centro de recreación deportiva conocido como el Hiram Bithorn y el Coliseo Roberto Clemente.

\section{LA PERCEPIÓN DEL ENTORNO URBANO}

Indudablemente la ciudad de San Juan es la manifestación de la restructuración del sistema productivo, de la globalización de su espacio y de sus relaciones socioeconónicas, así como el reflejo de la acumulación de nuevos patrones capitalistas y de modernas y sofisticadas tecnologias de comunicación. Aunque no negamos el carácter moderno de la ciudad, todavía queda mucho para hacer de San Juan una ciudad habitable donde coexistan desarrollo sostenible y calidad de vida apropiada.

En el mosaico Sanjuanero vemos como día a día la economía prevaleciente va fragmentando y segregando la población cada vez más. En un lado la clase media, en otro las clases marginadas compuestas por puertorriqueños y extranjeros indocumentados, en su mayoría dominicanos y en otro extremo la población de personas de un nivel económico bastante alto. El paisaje actual proyecta claramente una diferenciación social reflejando en cierta medida las condiciones sociales de sus habitantes. El resultado de este paisaje diferenciado pero por otro lado amalgamado es una serie de problemas que se acentúan cada vez más, como los problemas de suministros, contaminación, congestión vehicular, deterioro del paisaje, falta de vivienda apropiada, carencia de áreas verdes y servicios altamente estresados.

Utilizando el concepto de Hagget (1976) San Juan parece un pequeño universo estrellado, pueden distinguirse estrellas de muy distintos tamaños, a veces agrupadas en pequeñas constelaciones. A primera vista percibimos un caos en el conglomerado o en la mancha urbana. Pareciera no existir ningún orden. Pero lo cierto es que el orden urbano, lejos del precepto socialista del consumo equitativo de servicios, está en función de la economía capitalista de consumo. El criterio que prevalece es la fragmentación por sectores económicos.

¿Hacia dónde se dirige San Juan? Es hora de que el gobierno estatal y municipal se desprenda de su imágen paternalista de proveedor y provoque un desarrollo integrado y articulado en el entorno regional, bajo el 
paradigma ser humano-ambiente. La armonía y el equilibrio, valores intrinsecos a las corrientes culturalistas, deben insertarse en un modelo de desarrollo más homogéneo y sostenible (Precedo, 1996).

Las exigencias de sus habitantes van dirigidas a satisfacer las necesidades de la familia como el empleo, la vivienda y la educación. Las gestiones comunitarias en busca de un ambiente de calidad son cada vez más frecuentes. La seguridad fisica preocupa a sus habitantes por lo que el aumento en los niveles de criminalidad es inadmisible. La recreación social también es importante por lo que se debe hacer más presión por recuperar los parques y las plazas que permitan la convivencia social.

\section{UNA VISIÓN PEDAGÓGICA DE LA CIUDAD}

La ciudad es un gran laboratorio de estudio e investigación. A través de la lectura de su paisaje podemos estudiar las relaciones político-económicas y las relaciones sociales que emergen. En ella podemos encontrar el rastro de la huella histórica y el por qué de las decisiones que tomaron nuestros antepasados en torno a la organización del espacio. En la Carta de Atenas, Le Corbusier afirmó "que la historia se halla en los trazados y en las arquitecturas de los trazados. Lo que subsiste de los primeros constituye el hilo conductor, que junto con los textos y documentos gráficos, permite representar las sucesivas imágenes del pasado". Este espacio es un instrumento de trabajo para los que buscamos los porqués. La ciudad, en cada momento histórico, expresa o plasma, especialmente el tipo de sistema económico y social dominante. La variedad de manifestaciones urbanas no es por consiguiente, producto del azar, sino de las fuerzas políticas económicas, religiosas ... (Baigorri, 1987).

El trabajo de campo en la ciudad posiblemente cobre mayor importancia en la medida en que el tiempo avanza despavoridamente y los cambios que se sucitan son igual de violentos. Muchas veces no tenemos la oportunidad de conocer nuestro entorno sin que este haya cambiado y no nos hayamos dado cuenta. La percepción generalizada de la ciudad se toma hostil y violenta. Sin embargo, deberiamos buscar en ella, ya que es obra del ser humano armonia y equilibrio. Temas como la localización, la morfología, su origen, la población, el perfil socioeconómico y el uso de sus suelos se hacen imprescindibles.

El reto para los centros universitarios en Puerto Rico es cada día más interesante en cuanto a lo que ellos pueden aportar. A través de los pro- 
gramas de la Escuela de Planificación, la Facultad de Arquitectura, el Departamento de Geografía y el Departamento de Ciencias Ambientales de la Universidad de Puerto Rico asi, como de universidades privadas, se han promovido y gestado iniciativas para el desarrollo de planes y proyectos de concienciación y acción urbana. Pero todavía tenemos mucho trabajo que hacer con nuestro estudiantes, para hacerles entender que la ciudad no es un espacio vacío y deshumanizado. Nos toca buscar nuevos modelos de desarrollo donde prevalezca el equilibrio entre espacio y ser humano, entre ética y estética de manera que la ciudad de San Juan del futuro sea más habitable y humanistica

\section{REFERENCIAS}

BAIGORRI \& CASTÁN, (1987) "Enseñar la ciudad", De la Torre, Madrid.

BARALT, Guillermo.(1993) "Tradición de Futuro", Carimar, Puerto Rico.

DEL TORO, Alberto. (1 99 1) "Puerta de Tierra: tradición, historia y su camino al futuro' Planificación Ayer y Hoy", JP, PR.

GUTIÉRREZ PUEBLA, Javier.(1984) "La Ciudad y la Organización Pegional", Cincel, Barcelona

HARDOY, Jorge E. (1975). "La Forma de las ciudades coloniales en Hispanoamérica", Revista Internationale, \#5.

HERNANDO RICA, Agustin. (1990) "Hacia un Mundo de Ciudades", Cincel, Barcelona.

ANDREW. (1996), "The Urban Question" Cities, Arizona State University.

PRECEDO LEDO, Andrés. (1996). "Ciudad y Desarrollo Urbano", Sintesis, Madrid.(artículo inédito)

SÉPULVEDA RIVERA, Anibal.(1989) "San Juan: historia ilustrada de su desarrollo urbano, 1508-1898", Carimar. Puerto Rico.

ZÁRATE MARTIN , Antonio. (1999) "Globalización y Forma Urbana en el Caribe, el caso de San Juan de Puerto Rico" En América Latina: Lógicas Locales, y Lógicas Globales. coord. M Panadero F. Cebrián. Col. estudios Ediciones de la Universidad de Castilla- La Mancha, Ciudad Real.

ZORRILLA et. al.(1995) "El día que llegó el Huracán" En Puerto Rico:Vívencia y Querencia, Santillana, Puerto Rico

Plan de Ordenamiento Territorial del Municipio de San Juan, Fase Avanzada, Oficina de Urbanismo del Municipio de San Juan, 1999. 